\title{
Novel MRI and PET Markers of Neuroinflammation in Multiple Sclerosis
}

Marloes Hagens ${ }^{1}$, Bart van Berckel${ }^{2}$, Frederik Barkhof ${ }^{2}$

1. Department of Neurology and MS Center Amsterdam, VU University Medical Center, Neuroscience Campus Amsterdam, The Netherlands

2. Department of Radiology and Nuclear Medicine and MS Center Amsterdam, VU University Medical Center, Neuroscience Campus Amsterdam, The Netherlands

Correspondence to Professor Frederik Barkhof, Department of Radiology and Nuclear Medicine, VU University Medical Center, De Boelelaan 1118, 1081 HZ Amsterdam, The Netherlands. Tel: +31 20 444 0365; e-mail: f.barkhof@vumc.nl 


\section{ABSTRACT}

\section{Purpose of review}

Gadolinium-enhancement depicts BBB disruption associated with new inflammatory MRI lesions in MS and is widely used for diagnosis and therapeutic monitoring. However, earlier and more specific markers of inflammation are urgently needed.

\section{Recent findings}

Susceptibility Weighted Images demonstrate the importance of the central vein in the formation of MS lesions. Perfusion weighted imaging techniques can show focal and diffuse low-grade inflammatory changes not visible on conventional MRI. Leptomeningeal enhancement could be part of the etiology of subpial cortical MS lesions. Ultra-small Superparamagnetic Particles of Iron Oxide can identify neuroinflammatory changes in addition to gadolinium enhancement and as such identify different types and phases of MS lesions. TSPO PET-tracers identify activated microglia and an increase in TSPO uptake in both MS lesions and normal appearing brain tissue is related to disease severity and progression. A range of novel tracers for microglia activation are under development as well as radioligands that can label therapeutic drugs.

\section{Summary}

Novel MRI and PET techniques improve in vivo visualization and quantification of the pleomorphic aspects of neuroinflammation, providing us with a unique insight in its pathogenesis, clinical relevance and therapy responsiveness in MS.

\section{KEYWORDS}

multiple sclerosis, neuroinflammation, magnetic resonance imaging, positron emission tomography, 18kd-translocator protein TSPO 


\section{KEYPOINTS}

- Neuroinflammation in multiple sclerosis is a heterogeneous process that is inadequately visualized by conventional MRI.

- Novel MRI techniques such as Susceptibility Weighted Imaging and Perfusion Weighted Imaging provide us with new insights in the development of MS lesions and low-grade neuroinflammation.

- Novel contrast agent USPIO and new uses for traditional gadolinium based contrast agents identify different aspects of this pleomorphic process.

- $\quad$ TSPO PET shows that increased microglia activation is present in both normal appearing tissue and MS specific lesions and is related to disease severity and progression

- A wide range of novel PET markers will give us unique insights in the pathophysiology of MS and the pharmacokinetics of drugs used in this disease. 


\section{INTRODUCTION}

Multiple sclerosis (MS) is a complex immune-mediated disorder of the brain and spinal cord characterized by neuroinflammation, demyelination, gliosis, axonal degeneration and neuronal loss. During the past decades imaging techniques such as magnetic resonance imaging (MRI) have contributed considerably to the improvement of clinical decision making and the design of clinical trials in multiple sclerosis. Additionally, a range of novel tools using advanced MRI or positron emission tomography (PET) technologies have been emerging from which we can obtain unique insights into pathogenesis of MS. The present article will summarize current developments in MRI and PET techniques to depict neuroinflammation in MS.

\section{MAGNETIC RESONANCE IMAGING}

Conventional MRI techniques, such as T2- and T1-weighted sequences, are generally used in the diagnosis, follow-up and therapeutic consideration in individual MS patients and clinical trials. Currently the most commonly used MRI marker for acute inflammation is gadolinium enhancing white matter lesions (WML), indicating disrupted blood-brain barrier (BBB) in these active MS lesions. Gadolinium-based contrast agents (GBCA) are generally considered safe in patients without severe renal insufficiency, but there is a potential risk for anaphylactoid-type reactions.[1] Moreover, recent studies suggest accumulation of (potentially toxic) gadolinium may occur in brain tissue in the absence of any significant renal dysfunction. $[2 *, 3,4]$ Apart from the potential side effects of GBCA, the correlation between conventional MRI markers for neuroinflammation and the clinical manifestations of MS in an individual patient is modest. Histopathological studies have shown that (inflammatory) brain pathology in MS is much more diverse and widespread than identified with conventional MRI techniques alone. Various developments regarding in vivo MR imaging have advanced our understanding of the pleomorphic aspects of neuroinflammation in MS in recent years. 


\section{SUSCEPTIBILITY WEIGHTED IMAGES}

The magnetic property of brain tissue is largely determined by the presence of paramagnetic iron, oxygen levels of the blood in capillaries and veins and the amount of diamagnetic myelin and tissue water. Susceptibility Weighted Images (SWI) uses the differences in magnetic susceptibility in brain tissue to identify MS-related tissue changes such as iron deposition, myelin density and venous capillary network density. To provide a quantitative measure for susceptibility Quantitative Susceptibility Mapping (QSM) was developed. However, these techniques cannot disentangle the different and sometimes opposing factors that increase or decrease tissue susceptibility.[5] Considering the pathophysiology of neuroinflammatory lesions in MS, one of the most striking results from SWI studies is the identification of the central vein in lesions development. Such a central vein has been demonstrated in both periventricular and subcortical MS lesions and is significantly less frequent in non-MS WMLs.[6-8] Moreover, in a longitudinal 7 Tesla study by Dal-Bianco et al., an increase in volume of central veins of MS lesions was seen in the development of such a lesions.[9*] This underlines the primal role of the central vein in lesion formation. In agreement with this, a recent retrospective $3 \mathrm{~T}$ and $7 \mathrm{~T} \mathrm{MRI} \mathrm{study} \mathrm{by} \mathrm{Absinta} \mathrm{et} \mathrm{al.} \mathrm{demonstrated} \mathrm{subtle} \mathrm{signal} \mathrm{changes} \mathrm{on} \mathrm{T2-}$ FLAIR and T2* images up to 2 months before onset of focal gadolinium enhancing WML, that colocalize with the developing lesion's central vein.[10*] Finally, SWI images can depict areas with phase shift without signal increase in other sequences, which may reflect areas of microglia activation (pre-active lesions).[11]

\section{PERFUSION-WEIGHTED IMAGING}

Perfusion MRI is a collective term for three MR techniques that measure changes in cerebral hemodynamics. Dynamic contrast-enhanced (DCE) MRI measures the integrity of the BBB using GBCA as an in vivo marker of low-grade neuroinflammation. Increased permeability of the BBB due to neuroinflammation enables extravasation of GBCA and the extravascular accumulation of such contrast agents increases the signal intensity in T1-weighted MRI images. By repeated acquisition of 
T1-weighted images during infusion of GBCA, DCE-MRI reveals tissue properties at a microvascular level, providing quantitative information on BBB integrity and leakage space as measures of (lowgrade) neuroinflammation within and outside visible lesions.[12**,13]

Dynamic susceptibility contrast-enhanced (DSC) MRI measures cerebral blood volume and flow by monitoring the first pass of a bolus of GBCA by a series of T2*-weighted MRI images. Regional decreased in blood volume (CBV) and flow (CBF) have been reported in different stages of MS. $[14,15 *, 16]$

In contrast to the previous two methods, arterial spin labeling (ASL) is a non-invasive perfusion technique that uses magnetization of blood as an endogenous contrast agent. $\left[17^{*}, 18,19\right]$ In an ASL study using multiple delay times, an increase in bolus arrival time (BAT) was demonstrated, indicating reduced perfusion. This is suggested to be caused by widespread arteriolar vasodilation associated with neuroinflammation.[17*]

In various recent studies both an increase and a decrease in cerebral perfusion have been linked to neuro-inflammatory changes in MS and clinically isolated syndrome (CIS).[12**,13,14,15*,16,17*,18,19] Those contradictory results might imply changes in perfusion occur during inflammation. Standardization of acquisition techniques and post-processing methods could help further implementation of perfusion-weighted MRI.

\section{LEPTOMENINGEAL ENHANCEMENT}

Besides the parenchymal GBCA enhancement used in the diagnoses and therapeutic decision making in MS, leptomeningeal enhancement has been described as part of the pathophysiology of MS (Figure 1).[20] Similar to the process in lesions, gadolinium enhancement of the leptomeninges is a result of vascular leakage and an indirect measure of leptomeningeal inflammation. Traditionally leptomeningeal enhancement is considered suggestive of diagnoses other than MS (e.g. sarcoid), but pathological studies have associated such leptomeningeal inflammation with subpial cortical 
demyelination and neurodegeneration in both early and late phases of MS.[21,22] In the last year, two MRI studies have aimed to quantify the prevalence and distribution of leptomeningeal inflammation in MS using 3 Tesla post-contrast T2-weighted fluid-attenuated inversion recovery (T2FLAIR) MRI.[23**,24*] Absinta et al. reported focal, usually perivascular, leptomeningeal enhancement in 74 of 299 MS patients and in one of 37 neurologically healthy controls.[23**] Strikingly, this prevalence was nearly twice as frequent in progressive MS patients (33\%) compared to relapsing remitting MS (RRMS) (19\%). In two cases that came to autopsy and pathologic evaluation confirmed the presence of perivascular leptomeningeal inflammation in three foci that enhanced in vivo, all in close relation to confluent cortical demyelination. On the contrary, Eisele et al. reported only one single case of focal leptomeningeal enhancement in a cohort of $122 \mathrm{MS}$ patients of which only 15 had progressive MS.F[24*] As a positive control group, 5 patients with stroke where included, all of whom showed leptomeningeal enhancement in a diffuse pattern. The conspicuous difference in prevalence between both studies could be attributed to the differences in patient population or in scanning techniques. This argues for further studies to determine the actual prevalence, natural history and clinical relevance of focal leptomeningeal enhancement in MS and its relation to cortical lesion formation.

\section{ULTRASMALL SUPERPARAMAGNETIC PARTICLES OF IRON OXIDE}

As mentioned before, gadolinium-enhancement indicates a defective BBB and is therefore an indirect measure of neuroinflammation in MS. In these lesions infiltrating blood-born monocytes play a crucial role, causing myelin breakdown and phagocytosing myelin debris. The phagocytic infiltration in the CNS can be directly detected in vivo with the use of Ultra-small Superparamagnetic Particles of Iron Oxide (USPIO) as an MRI contrast agent. Several hours after intravenous administration of USPIO these small nanoparticles are captured by phagocytic monocytes and accumulate in these cells.[25] Their iron oxide core distorts the magnetic field, causing a rapid decreases in T1- and T2-relaxation times of water molecules, resulting in an increased signal intensity on T1-weighted images and a 
decrease of signal on T2-weighted-gradient echo images.[25,26] Areas of altered intensity on delayed MRI scans presumably reflect the infiltration of USPIO-loaded macrophages in active MS lesions. USPIO enhancement can have one of three patterns: focal, ring-like or "return-to-isointensity" (after having been hypointense on native T1-weighted images).[27] Furthermore, global changes in the T1 signal in normal appearing brain tissue can demonstrate subtle generalized inflammatory activity without apparent BBB damage.[28]

Clinical pilot trials have demonstrated that the use of both GBCA and USPIO in MS patients identifies more inflammatory lesions than gadolinium alone, range from a $4 \%$ to $244 \%$ increase. $[27,29,30]$ The subgroup of lesions that enhanced with both contrast agents were characterized by a more severe evolution.[27,30]

A recent study by Maarouf et al. in $18 \mathrm{CIS}$ patients showed that USPIO enhancing lesions are already present at the onset of MS, but to a lesser extent than previously reported in RRMS patients.[31*] The presence of such lesions was associated with more severe and persistent local tissue injury after 12 months of follow-up, but not with changes in Expanded Disability Status Scale (EDSS). These limited number of studies show that USPIO allow for measurement of inflammation not possible with other MRI contrast agents, but the lack of available contrast-agents limits further development.

\section{POSITRON EMISSION TOMOGRAPHY}

PET is a noninvasive molecular imaging technique to quantify biochemical and physiological processes in vivo. It measures the bio-distribution of a radioligand or tracer, a radioactive isotope bound to biologically active molecule. Over the last decades more and more new radioligands have been developed, creating a unique insight in the pathophysiology of neuroinflammation, neuronal dysfunction, demyelination and remyelination. In this review we will focus on PET-tracers as biomarkers for neuroinflammation. 


\section{IMAGING ACTIVATED MICROGLIA}

The complex and highly dynamic processes of microglia activation is the central characteristic of neuroinflammation and a key element of neurodegeneration in MS.[32,33] Initially, in vivo PETstudies investigating microglia activation used radioligands that bind to the $18 \mathrm{kd}$-translocator protein (TSPO), formerly known as peripheral benzodiazepine receptor. The first successful and now most frequently used of the TSPO-radioligands is $\left[{ }^{11} \mathrm{C}\right]$ PK11195.[34] $\left[{ }^{11} \mathrm{C}\right]$ PK11195 uptake is increased in focal T2 MRI lesions, co-localizes with gadolinium-enhancing T1 lesions in RRMS and is increased in normal appearing white and grey matter (NAWM and NAGM) in MS patients compared to healthy controls.[35-37] Overall there is significant positive correlation with $\left[{ }^{11} \mathrm{C}\right] \mathrm{PK} 11195$ uptake and disease duration, disability score and brain atrophy.[35-38] However, $\left[{ }^{11} \mathrm{C}\right] \mathrm{PK} 11195$ has several disadvantages, including limited brain entrance, poor signal-to-noise ratio and labeling with the impractically rapid decaying isotope $\left[{ }^{11} \mathrm{C}\right]\left(\mathrm{t}_{1 / 2}=20.33\right.$ minutes).[39] This led to the development of a wide range of second generation TSPO radioligands, see table 1 for an overview of TSPO PET-studies in MS patients. The main disadvantage of these new tracers is a single nucleotide polymorphisms in the TSPO gene (rs697) that induces a variation in binding activity for these second generation TSPO tracers.[50] Differences in affinity between the high-affinity binding type and the low-affinity binding type varies between the different TSPO tracers from a 4-fold to as high as a 50 -fold decrease in binding.[50]

Besides the use of different tracers and genetic polymorphism, comparison of TSPO PET studies can be challenging due to differences in quantification methods of specific binding, as well as differences in patient population, such as subtypes of MS, disease duration, disease severity and age. Recent studies have illustrated the relevance of imaging these different subgroups in further understanding the pathophysiology of the different disease stages in MS.

Giannetti et al. recruited eighteen CIS patients for a $\left[{ }^{11} \mathrm{C}\right]$ PK11195 study.[43*] They demonstrated a global increase in $\left[{ }^{11} \mathrm{C}\right] \mathrm{PK} 11195$ uptake in NAWM and deep grey matter compared to healthy controls 
and a further significant increase in those CIS patients with T2 MRI lesions. Furthermore, higher levels of uptake was correlated with a development of MS at 2 year interval. This suggests that diffuse inflammatory changes in the NAWM in early phases of the disease predispose for WML development and subsequently conversion to MS. This would be in line with previous ex vivo immunehistochemical studies, showing areas of activated microglia without apparent loss of myelin at risk of developing into acute inflammatory lesions.[35,51,52]

Rissanen et al. used the same TSPO-tracer to image not early but chronic disease in the secondary progressive MS (SPMS) patients.[41*] First of all they showed an increase in $\left[{ }^{11} \mathrm{C}\right]$ PK11195 uptake in $57 \%$ of black holes, which has recently been related to a higher degree of clinical disability by Giannetti et al.[42*] Moreover, there was significant increase in tracer uptake in the global NAWM and in the thalamus in SPMS patients compared to healthy controls. This is in agreement with the pathology findings of a more chronic and diffuse low-grade inflammation behind the BBB in the progressive phase of the disease.[53]

In the RRMS there seems to be an association between disease activity and global microglia activity measured with TSPO PET. On one hand, Park et al. found no increase in $\left[{ }^{11} \mathrm{C}\right]$ PBR28 uptake in the NAWM of four clinically and radiologically stable RRMS patients.[45*] However, Colasanti et al. described a positive relation between $\left[{ }^{18} \mathrm{~F}\right]$ PBR111 uptake and disease severity scores in eleven RRMS patients.[46*]

Overall, increase in TSPO uptake appears to be related to disease severity and progression.

\section{NOVEL PET-TRACERS FOR ACTIVATED MICROGLIA}

Besides TSPO radioligands, new PET-tracers with different binding targets on microglia are under development.

In MS the purinergic, adenosine triphosphate (ATP) binding, receptor P2X7 (P2X7R) is upregulated on microglia. Binding of ATP to the receptor activates the microglia, which leads to proliferation and 
release of the pro-inflammatory cytokine and subsequently microglia recruitment.[54] Recently, ATPdependent release of tumor necrosis factor $\alpha$ (TNF $\alpha$ ) was demonstrated to have a neuroprotective effect in MS.[55] Moreover, a rare single nucleotide polymorphism rs28360457 in the P2X7R gene has been described to protect against the risk of developing MS.[56*] All in all, P2X7R is an interesting target for PET-tracers and potentially also for drug development. A first P2X7R PET-tracer [11C]A-740003 is under evaluation in animal models of neuroinflammation by Janssen et al.[57] Gao et al. developed a second radioligand [11C]GSK1482160 which has yet to be evaluated in vivo.[58]

Secondly, the adenosine A2Areceptor (A2AR) has been proposed as a new in vivo target for imaging neuro-inflammation. In vitro studies show inflammatory stimuli lead to upregulation of A2AR on microglial cells and subsequently alter their morphology and behavior.[59] This possibly provides an endogenous pathway to limit neuro-inflammation and alter the pathophysiology process of neurodegeneration. [60] Rissanen et al. showed an increase uptake of the A2AR PET-tracer $\left[{ }^{11} \mathrm{C}\right]$ TMSX in NAWM of MS patients compared to healthy controls, which was associated with higher EDSS and increased brain tissue loss.[61,62] On the downside, $\left[{ }^{11} \mathrm{C}\right]$ TMSX suffers from low binding potentials and high non-specific binding. Therefore, new A2AR tracers are currently under investigations.

\section{LABELLING DRUGS THAT TARGET NEUROINFLAMMATION}

Furthermore, PET-tracers have been developed to analyze the organ and tissue distribution of drugs currently used in or under development for treatment of MS. As these drugs usually have a long pharmacokinetic half-life the long-lived positron emitters $\left[{ }^{124} \mathrm{l}\right]\left(\mathrm{t}_{1 / 2}=4.2\right.$ days $)$ and $\left[{ }^{89} \mathrm{Zr}\right]\left(\mathrm{t}_{1 / 2}=3.3\right.$ days) are more commonly used.

To quantify the brain penetration of siponimod (BAF312), a selective sphingosine-1-phosphate receptor (S1PR) agonist in phase 3 development for treatment of SPMS, Briard et al. developed 
compound MS565.[63,64] By slightly modifying the structure of BAF312 this molecule can be labelled to $\left[{ }^{124} \mathrm{I}\right]$ for PET imaging. Despite the additional iodine atom the biochemical properties and pharmacokinetics of $\left[{ }^{124} \mathrm{I}\right] \mathrm{MS} 565$ remain very close to the original compound. In a similar fashion they previously developed $\left[{ }^{124}\right.$ I]BZM055 as a surrogate tracer to study the biodistribution of fingolimod (FTY720), a S1PR agonist approved for the treatment of RRMS.[65] Further validation of these radioligands is necessary.

In contrast to radioligands based on MS specific small molecules, radioactive labelling of monoclonal antibodies (mAbs) has already been validated in oncology.[66] Similar studies in MS patients can give us important insights in the mechanisms responsible for the therapeutic effect of mAbs in MS. A pilot study presented at ECTRIMS 2015 suggested that $\left[{ }^{89} \mathrm{Zr}\right.$ ]-labelled rituximab, a chimeric monoclonal antibody (mAb) against the CD20 molecule on mature B lymphocytes used in the treatment of RRMS.[67] There was no evidence of cerebral penetration for rituximab in this pilot study with two active RRMS patients, suggesting the (early) therapeutic effects of this drug are independent of CNS penetration. Further larger studies with labeled mAbs are highly relevant, especially in light of the recently reported phase III result of the ocrelizumab trials in MS.[68]

\section{CONCLUSION}

Current advances in MRI and PET techniques have expanded the armamentarium for in vivo visualization and quantification of the pleomorphic aspects of neuroinflammation in MS, providing us with a unique insight in its pathogenesis, clinical relevance and therapy responsiveness not possible with conventional MRI techniques. Further work should aim at validating these novel imaging methods and ideally implementing them in the clinical practice and drug development. 


\section{Acknowledgements}

The authors wish to thank dr J. Killestein, prof. dr. A.D. Windhorst, prof. dr. A.A. Lammertsma, dr. A.M.W. van Dam, prof. dr. H.E. de Vries for their help on various projects.

\section{Financial support and sponsorship}

This work was supported by MS Center Amsterdam and Neuroscience Campus Amsterdam.

\section{Conflicts of interest}

M.H. has no conflicts of interest.

B.v.B. has no conflicts of interest.

F.B. serves on the editorial boards of Brain, Neurology, Neuroradiology, Multiple Sclerosis Journal and Radiology, and serves as a consultant for Bayer-Schering Pharma, Sanofi-Aventis, Genzyme, Biogen-Idec, Teva, Novartis, Roche, Synthon BV and Jansen Research. 


\section{REFERENCES AND RECOMMENDED READING}

1. Khawaja AZ, Cassidy DB, Al Shakarchi J, et al.: Revisiting the Risks of MRI with Gadolinium Based Contrast Agents-review of Literature and Guidelines. Insights Imaging 2015, 6:553558.

*2. Kanda T, Ishii K, Kawaguchi H, et al.: Hyperintensity in Dentate Nucleus and Globus Pallidus on Unenhanced MR Images Kanda et Al. Radiology 2014, 270:834-841.

This is the first study to describe a cumulative effect of previous GBCA administrations on the signal intensity in the dentate nucleus and globus pallidus of unenhanced T1-weighted MR images.

3. Errante $\mathrm{Y}$, Cirimele V, Mallio CA, et al.: Progressive Increase of T1 Signal Intensity of the Dentate Nucleus on Unenhanced Magnetic Resonance Images Is Associated With Cumulative Doses of Intravenously Administered Gadodiamide in Patients With Normal Renal Function, Suggesting Dechelation. Invest. Radiol. 2014, 49:685-690.

4. McDonald RJ, McDonald JS, Kallmes DF, et al.: Intracranial Gadolinium Deposition after Contrast-Enhanced MR Imaging. Radiology 2015, 275:772-82.

5. Liu C, Li W, Tong K a, et al.: Susceptibility-Weighted Imaging and Quantitative Susceptibility Mapping in the Brain. J. Magn. Reson. imaging 2015, 42:23-41.

6. Kau T, Taschwer M, Deutschmann H, et al.: The "Central Vein Sign": Is There a Place for Susceptibility Weighted Imaging in Possible Multiple Sclerosis? Eur. Radiol. 2013, 23:19561962.

7. Maggi P, Mazzoni LN, Moretti M, et al.: SWI Enhances Vein Detection Using Gadolinium in Multiple Sclerosis. Acta radiol. 2015, 4:1-4.

8. Lane JI, Bolster B, Campeau NG, et al.: Characterization of Multiple Sclerosis Plaques Using Susceptibility-Weighted Imaging at 1.5 T: Can Perivenular Localization Improve Specificity of Imaging Criteria? J. Comput. Assist. Tomogr. 2015, 39:317-320.

*9. Dal-Bianco A, Hametner S, Grabner G, et al.: Veins in Plaques of Multiple Sclerosis Patients - a Longitudinal Magnetic Resonance Imaging Study at 7 Tesla. Eur. Radiol. 2015.

This 7T longitudinal study using SWI shows the volume of intralesional veins is significantly increased during the development of MS lesions, underlining the primal role of the central vein in lesion formation.

*10. Absinta M, Nair G, Sati P, et al.: Direct MRI Detection of Impending Plaque Development in Multiple Sclerosis. Neurol. Neuroimmunol. Neuorinflammation 2015, 2:e145.

Subtle signal changes on T2-FLAIR and T2* images can be seen up to 2 months before onset of focal gadolinium enhancing WML.

11. Kakeda S, Futatsuya K, Ide S, et al.: Improved Detection of Cortical Gray Matter Involvement in Multiple Sclerosis with Quantitative Susceptibility Mapping. Acad. Radiol. 2015, 22:14271432. 
**12. Cramer SP, Simonsen H, Frederiksen JL, et al.: Abnormal Blood-Brain Barrier Permeability in Normal Appearing White Matter in Multiple Sclerosis Investigated by MRI. Neurolmage Clin. 2014, 4:182-9.

Permeability of the BBB in peri-ventricular NAWM measured by DCE-MRI, can significantly improve prediction of conversion to MS in optic neuritis patients, compared to T2 lesion count alone.

13. Cramer SP, Modvig S, Simonsen HJ, et al.: Permeability of the Blood - Brain Barrier Predicts Conversion from Optic Neuritis to Multiple Sclerosis. Brain 2015, 138:2571-2583.

14. Sowa P, Bjørnerud A, Nygaard GO, et al.: Reduced Perfusion in White Matter Lesions in Multiple Sclerosis. Eur. J. Radiol. 2015, 84:2605-2612.

*15. Bester M, Forkert ND, Stellmann JP, et al.: Increased Perfusion in Normal Appearing White Matter in High Inflammatory Multiple Sclerosis Patients. PLoS One 2015, 10.

DSC-MRI is sensitive to diffuse microvascular pathology in NAWM and this differentiates between high- and low-inflammatory patients.

16. Papadaki EZ, Simos PG, Panou T, et al.: Hemodynamic Evidence Linking Cognitive Deficits in Clinically Isolated Syndrome to Regional Brain Inflammation. Eur. J. Neurol. Neurol 2014, 21:499-505.

*17. Paling D, Thade Petersen E, Tozer DJ, et al.: Cerebral Arterial Bolus Arrival Time Is Prolonged in Multiple Sclerosis and Associated with Disability. J. Cereb. Bood Flow Metab. 2014, 34:34-42.

This ASL MRI study shows altered cerebral aterial haemodynamics in NAWM and deep grey matter is related to clinical disability scores, possibly resulting from widespread inflammaory changes.

18. Narayana P a, Zhou Y, Hasan KM, et al.: Hypoperfusion and T1-Hypointense Lesions in White Matter in Multiple Sclerosis. Mult. Scler. J. 2014, 20:365-73.

19. Debernard L, Melzer TR, Van Stockum S, et al.: Reduced Grey Matter Perfusion without Volume Loss in Early Relapsing-Remitting Multiple Sclerosis. J. Neurol. Neurosurg. Psychiatry 2014, 85:544-551.

20. Guseo A, Jellinger K: The Significance of Perivascular Infiltrations in Multiple Sclerosis. J. Neurol. 1975, 211:51-60.

21. Lucchinetti CF, Popescu BFG, Bunyan RF, et al.: Inflammatory Cortical Demyelination in Early Multiple Sclerosis. N. Engl. J. Med. 2011, 365:2188-2197.

22. Choi SR, Howell OW, Carassiti D, et al.: Meningeal Inflammation Plays a Role in the Pathology of Primary Progressive Multiple Sclerosis. Brain 2012, 135:2925-2937.

**23. Absinta M, Vuolo L, Rao A, et al.: Gadolinium-Based MRI Characterization of Leptomeningeal Inflammation in Multiple Sclerosis. Neurology 2015, 85:18-28.

This study demonstrates a rather high prevalence of leptomeningeal enhancement in especially progressive MS patients. The association with cortical MS lesions is highly relavent in understanding the pathophysiology of such lesions. 
*24. Eisele P, Griebe M, Szabo K, et al.: Investigation of Leptomeningeal Enhancement in MS: A Postcontrast FLAIR MRI Study. Neurology 2015, 84:770-775.

Reporting a very low prevalence of leptomeningeal enhancement in MS in contrary to the previous reference, this article creates an interesting discussion on the importance of leptomeningeal enhancement in the pathophysiology of MS.

25. Dousset V, Ballarino L, Delalande C, et al.: Comparison of Ultrasmall Particles of Iron Oxide (USPIO)-Enhanced T2-Weighted, Conventional T2-Weighted, and Gadolinium-Enhanced T1Weighted MR Images in Rats with Experimental Autoimmune Encephalomyelitis. Am. J. Neuroradiol. 1999, 20:223-227.

26. Corot C, Robert P, Idée JM, et al.: Recent Advances in Iron Oxide Nanocrystal Technology for Medical Imaging. Adv. Drug Deliv. Rev. 2006, 58:1471-1504.

27. Vellinga MM, Oude Engberink RD, Seewann A, et al.: Pluriformity of Inflammation in Multiple Sclerosis Shown by Ultra-Small Iron Oxide Particle Enhancement. Brain 2008, 131:800-807.

28. Vellinga MM, Vrenken $\mathrm{H}$, Hulst $\mathrm{HE}$, et al.: Use of Ultrasmall Superparamagnetic Particles of Iron Oxide (USPIO)-Enhanced Mri to Demonstrate Diffuse Inflammation in the NormalAppearing White Matter (NAWM) of Multiple Sclerosis (MS) Patients: An Exploratory Study. J. Magn. Reson. Imaging 2009, 29:774-779.

29. Dousset V, Brochet B, Deloire MS a, et al.: MR Imaging of Relapsing Multiple Sclerosis Patients Using Ultra-Small-Particle Iron Oxide and Compared with Gadolinium. Am. J. Neuroradiol. Neuroradiol. 2006, 27:1000-1005.

30. Tourdias T, Stéphanie Roggerone M, Massimo Filippi M, et al.: Assessment of Disease Activity in Multiple Sclerosis Phenotypes with Combined Gadolinium- and Superparamagnetic Iron Oxide-enhanced MR Imaging. Radiology 2012, 264:225-233.

*31. Maarouf A, Ferre JC, Zaaraoui W, et al.: Ultra-Small Superparamagnetic Iron Oxide Enhancement Is Associated with Higher Loss of Brain Tissue Structure in Clinically Isolated Syndrome. Mult. Scler. J. 2015 [Epub ahead of print]

As the first USPIO study published in a number of years demonstrates macrophage infiltration early on in the disease and its relation to the severity of neuroinflammatory changes in the brain.

32. Hemmer B, Kerschensteiner $\mathrm{M}$, Korn $\mathrm{T}$ : Role of the Innate and Adaptive Immune Responses in the Course of Multiple Sclerosis. Lancet Neurol. 2015, 14:406-419.

33. Mahad DH, Trapp BD, Lassmann H: Pathological Mechanisms in Progressive Multiple Sclerosis. Lancet Neurol. 2015, 14:183-193.

34. Vowinckel E, Reutens D, Becher B, et al.: PK11195 Binding to the Peripheral Benzodiazepine Receptor as a Marker of Microglia Activation in Multiple Sclerosis and Experimental Autoimmune Encephalomyelitis. J. Neurosci. Res. 1997, 50:345-353.

35. Banati RB, Newcombe J, Gunn RN, et al.: The Peripheral Benzodiazepine Binding Site in the Brain in Multiple Sclerosis: Quantitative in Vivo Imaging of Microglia as a Measure of Disease Activity. Brain 2000, 123:2321-37. 
36. Debruyne JC, Versijpt J, Laere van KJ, et al.: PET Visualization of Microglia in Multiple Sclerosis Patients Using [ 11 C]PK11195. Eur. J. Neurol. 2003, 10:257-264.

37. Politis M, Giannetti P, Su P, et al.: Increased PK11195 PET Binding in the Cortex of Patients with MS Correlates with Disability. Neurology 2012, 79:523-30.

38. Versijpt J, Debruyne JC, Laere van KJ, et al.: Microglial Imaging with Positron Emission Tomography and Atrophy Measurements with Magnetic Resonance Imaging in Multiple Sclerosis: A Correlative Study. Mult. Scler. J. 2005, 11:127-134.

39. Kreisl WC, Fujita M, Fujimura Y, et al.: Comparison of [11C]-(R)-PK 11195 and [11C]PBR28, Two Radioligands for Translocator Protein (18 kDa) in Human and Monkey: Implications for Positron Emission Tomographic Imaging of This Inflammation Biomarker. Neuroimage 2010, 49:2924-2932.

40. Ratchford JN, Endres CJ, Hammoud DD, et al. Decreased microglial activation in MS patients treated with glatiramer acetate. J. Neurol. 2002, 259:1199-205.

*41. Rissanen E, Tuisku J, Rokka J, et al.: In Vivo Detection of Diffuse Inflammation in Secondary Progressive Multiple Sclerosis Using PET Imaging and the Radioligand 11 C-PK11195. J. Nucl. Med. 2014, 55:939-944.

This in vivo TSPO PET study supports earlier neurpathological studies indentifying the pivotal role of microglia in low-grade inflammation behind intact BBB in the SPMS.

*42. Giannetti P, Politis M, Su P, et al.: Microglia Activation in Multiple Sclerosis Black Holes Predicts Outcome in Progressive Patients: An in Vivo [(11)C](R)-PK11195-PET Pilot Study. Neurobiol. Dis. 2014, 65:203-210.

A heterogenous binding pattern of $\left[{ }^{11} \mathrm{C}\right] \mathrm{PK} 11195$ in black holes related to the severity of the disease course in progressive MS, indicates clinically relevant inflammatory activity in these "holes".

**43. Giannetti P, Politis M, Su P, et al.: Increased PK11195-PET Binding in Normal-Appearing White Matter in Clinically Isolated Syndrome. Brain 2015, 138:110-119.

This is a first TSPO PET study in CIS patients. It identifies diffuse inflammatory changes in the NAWM that predispose for WML development and subsequently conversion to MS.

44. Oh U, Fujita M, Ikonomidou VN, et al. Translocator protein PET imaging for glial activation in multiple sclerosis. J. Neuroimmune Pharmacol. 2011, 6:354-361.

*45. Park E, Gallezot J-D, Delgadillo A, et al.: 11C-PBR28 Imaging in Multiple Sclerosis Patients and Healthy Controls: Test-Retest Reproducibility and Focal Visualization of Active White Matter Areas. Eur. J. Nucl. Med. Mol. Imaging 2015, 42:1081-1092.

This test-retest study demonstrates the reliability of the second generation TSPO radioligand $\left[{ }^{11} \mathrm{C}\right] \mathrm{PBR} 28$ in MS.

*46. Colasanti A, Guo Q, Muhlert N, et al.: In Vivo Assessment of Brain White Matter Inflammation in Multiple Sclerosis with [18F]-PBR111 PET. J. Nucl. Med. 2014, 55:1112-1118. 
This is a first study using the second generation TSPO tracer $\left[{ }^{18} \mathrm{~F}\right] \mathrm{PBR} 111$ in MS patients. It shows a positive correlation between $\left[{ }^{18} \mathrm{~F}\right] \mathrm{PBR} 111$ uptake and MS severity scores in RRMS.

47. Vas Á, Shchukin Y, Karrenbauer VD, et al. Functional neuroimaging in multiple sclerosis with radiolabelled glia markers: Preliminary comparative PET studies with [11C]vinpocetine and [11C]PK11195 in patients. J. Neurol. Sci. 2008, 264:9-17 .

48. Takano A, Piehl F, Hillert J, et al. In vivo TSPO imaging in patients with multiple sclerosis: a brain PET study with [18F]FEDAA1106. Eur. J. Nucl. Med. Mol. Imaging Res. 2013, 3:30.

49. Trigg W, Buckley C, Heurling K, et al. Evaluation of [18F]GE-180 PET imaging in relapsingremitting multiple sclerosis patients: a first-in-human study. ECTRIMS Online Libr. 2015.

50. Owen DRJ, Gunn RN, Rabiner EA, et al.: Mixed-Affinity Binding in Humans with 18-kDa Translocator Protein Ligands. J. Nucl. Med. 2011, 52:24-32.

51. Marik C, Felts P a., Bauer J, et al.: Lesion Genesis in a Subset of Patients with Multiple Sclerosis: A Role for Innate Immunity? Brain 2007, 130:2800-2815.

52. Van Horssen J, Singh S, van der Pol S, et al.: Clusters of Activated Microglia in NormalAppearing White Matter Show Signs of Innate Immune Activation. J. Neuroinflammation 2012, 9:156.

53. Allen I V, McQuaid S, Mirakhur M, et al.: Pathological Abnormalities in the Normal-Appearing White Matter in Multiple Sclerosis. Neurol. Sci. 2001, 22:141-144.

54. Monif M, Burnstock G, Williams D a.: Microglia: Proliferation and Activation Driven by the P2X7 Receptor. Int. J. Biochem. Cell Biol. 2010, 42:1753-1756.

55. Masuch A, Shieh C-H, van Rooijen N, et al.: Mechanism of Microglia Neuroprotection: Involvement of P2X7, TNFa, and Valproic Acid. Glia 2016, 64:76-89.

*56. Gu BJ, Field J, Dutertre S, et al.: A Rare P2X7 Variant Arg307GIn with Absent Pore Formation Function Protects against Neuroinflammation in Multiple Sclerosis. Hum. Mol. Genet. 2015, 24:5644-5654.

A rare loss of function SNP in the P2X7 gene has a 1.8 fold protective effect on the risk of developing $\mathrm{MS}$ in a large Australian and a large European cohort.

57. Janssen B, Vugts DJ, Funke $U$, et al.: Synthesis and Initial Preclinical Evaluation of the P2X7 Receptor Antagonist [11C]A-740003 as a Novel Tracer of Neuroinflammation. J. Label. Compd. Radiopharm. 2014, 57:509-516.

58. Gao M, Wang M, Green M a., et al.: Synthesis of [11C]GSK1482160 as a New PET Agent for Targeting P2X7 Receptor. Bioorg. Med. Chem. Lett. 2015, 25:1965-1970.

59. Orr AG, Orr AL, Li X-J, et al.: Adenosine A2A Receptor Mediates Microglial Process Retraction. Nat. Neurosci. 2009, 12:872-878.

60. Santiago AR, Baptista FI, Santos PF, et al.: Role of Microglia Adenosine A(2A) Receptors in Retinal and Brain Neurodegenerative Diseases. Mediators Inflamm. 2014, 2014. 
61. Rissanen E, Virta JR, Paavilainen T, et al.: Adenosine A2A Receptors in Secondary Progressive Multiple Sclerosis: A [(11)C]TMSX Brain PET Study. J. Cereb. blood flow Metab. 2013, 33:1394-401.

62. Rissanen E, Tuisku J, Luoto P, et al.: Automated Reference Region Extraction and PopulationBased Input Function for Brain [(11)C]TMSX PET Image Analyses. J. Cereb. Bood Flow Metab. 2015, 35:157-165.

63. Briard E, Rudolph B, Desrayaud S, et al.: MS565: A SPECT Tracer for Evaluating the Brain Penetration of BAF312 (Siponimod). ChemMedChem 2015, 10:1008-1018.

64. Tavares A, Barret O, Alagille D, et al.: Brain Distribution of MS565, an Imaging Analogue of Siponimod (BAF312), in Non-Human Primates. Neurology 2014, 82:P1.168.

65. Briard E, Orain D, Beerli C, et al.: BZM055, an lodinated Radiotracer Candidate for PET and SPECT Imaging of Myelin and FTY720 Brain Distribution. ChemMedChem 2011, 6:667-677.

66. Wright BD, Lapi SE: Designing the Magic Bullet? The Advancement of Immuno-PET into Clinical Use. J. Nucl. Med. 2013, 54:1171-4.

67. Hagens MHJ, Killestein J, Dongen GAMS van, et al.: Imaging of Rituximab-Zirconium-89 Uptake with PET Scans in Active Relapsing-Remitting MS Patients. ECTRIMS Online Libr. 2015.

68. Montalban X, Hemmer B, Rammohan K, et al.: Efficacy and Safety of Ocrelizumab in Primary Progressive Multiple Sclerosis - Results of the Placebo-Controlled, Double-Blind, Phase III ORATORIO Study. ECTRIMS Online Libr. 2015. 
Figure 1 Leptomeningeal enhancement in MS

Post-contrast sagittal and axial 3D fluid attenuated inversion recovery (FLAIR) images obtained at 3 Tesla showing focal leptomeningeal enhancement in two relapsing remitting MS patients clinically and radiologically stable under (A) interferon beta 1-alpha and (B) glatiramer acetate treatment.

(Original figure)

Table 1 Overview of in vivo positron emission tomography studies with 18kd-translocator protein (TSPO) tracers in multiple sclerosis patients.

$\mathrm{CIS}=$ clinically isolated syndrome, EDSS = expanded disability status scale, GM = grey matter, $\mathrm{HC}=$ healthy control, $\mathrm{MS}=$ multiple sclerosis, $\mathrm{NAWM}=$ normal appearing white matter, $\mathrm{PPMS}=$ primary progressive multiple sclerosis, RRMS = relapsing remitting multiple sclerosis, SPMS = secondary progressive multiple sclerosis, $\mathrm{WM}=$ white matter, $\mathrm{WML}=$ white matter lesions

(Original Table) 


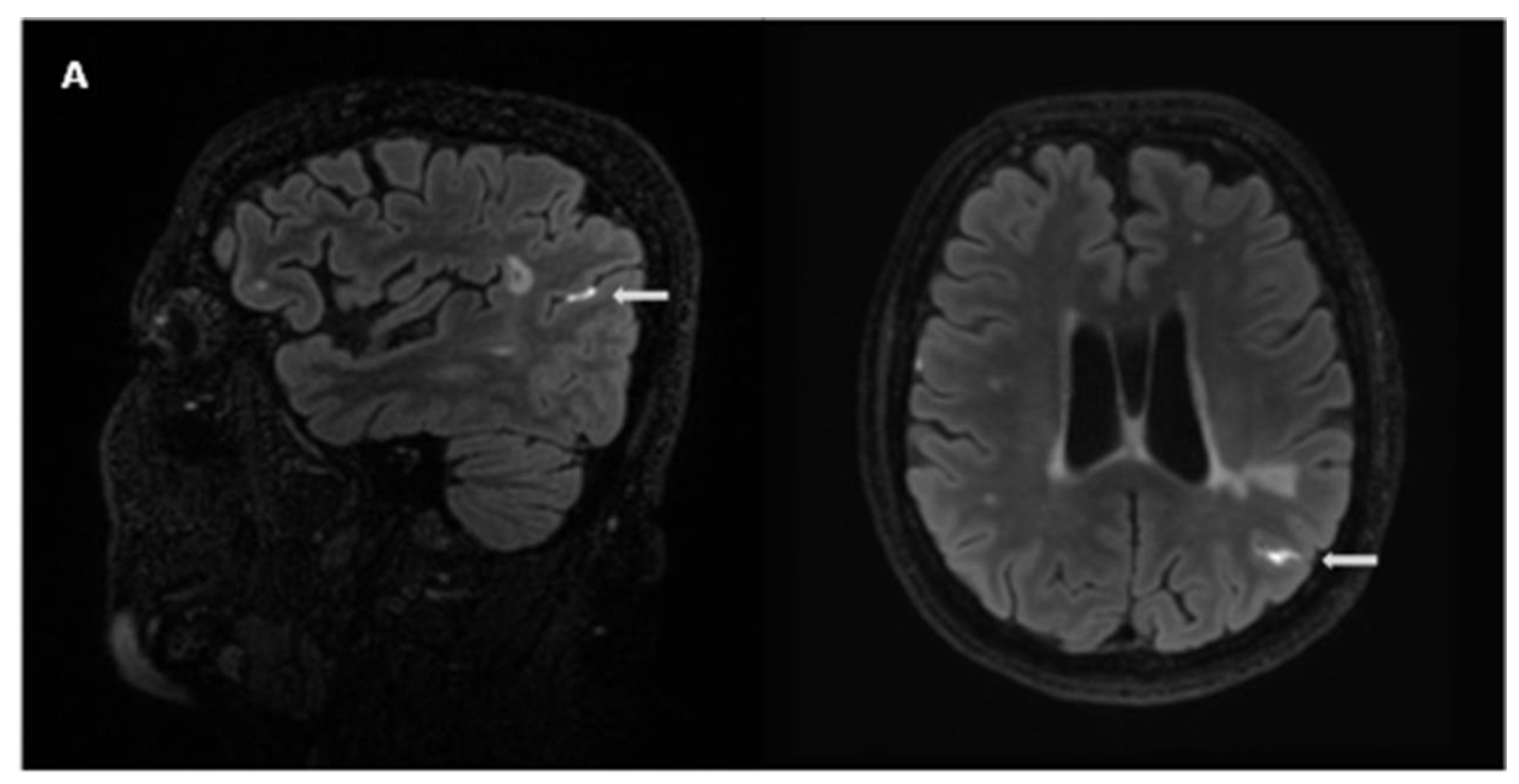

B
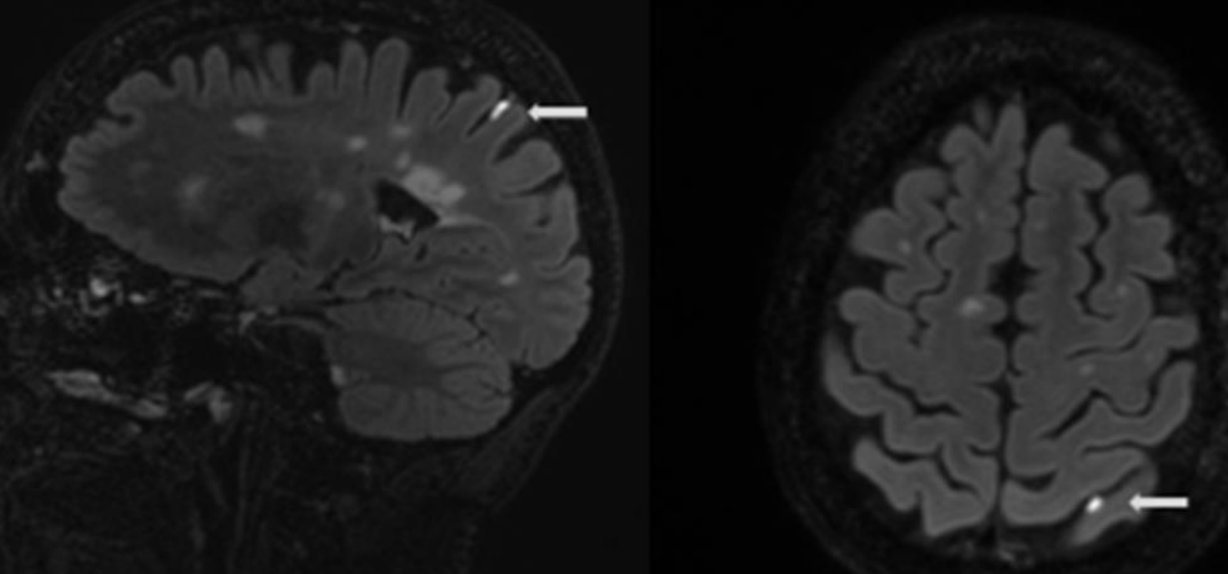
Tabel 1 (original)

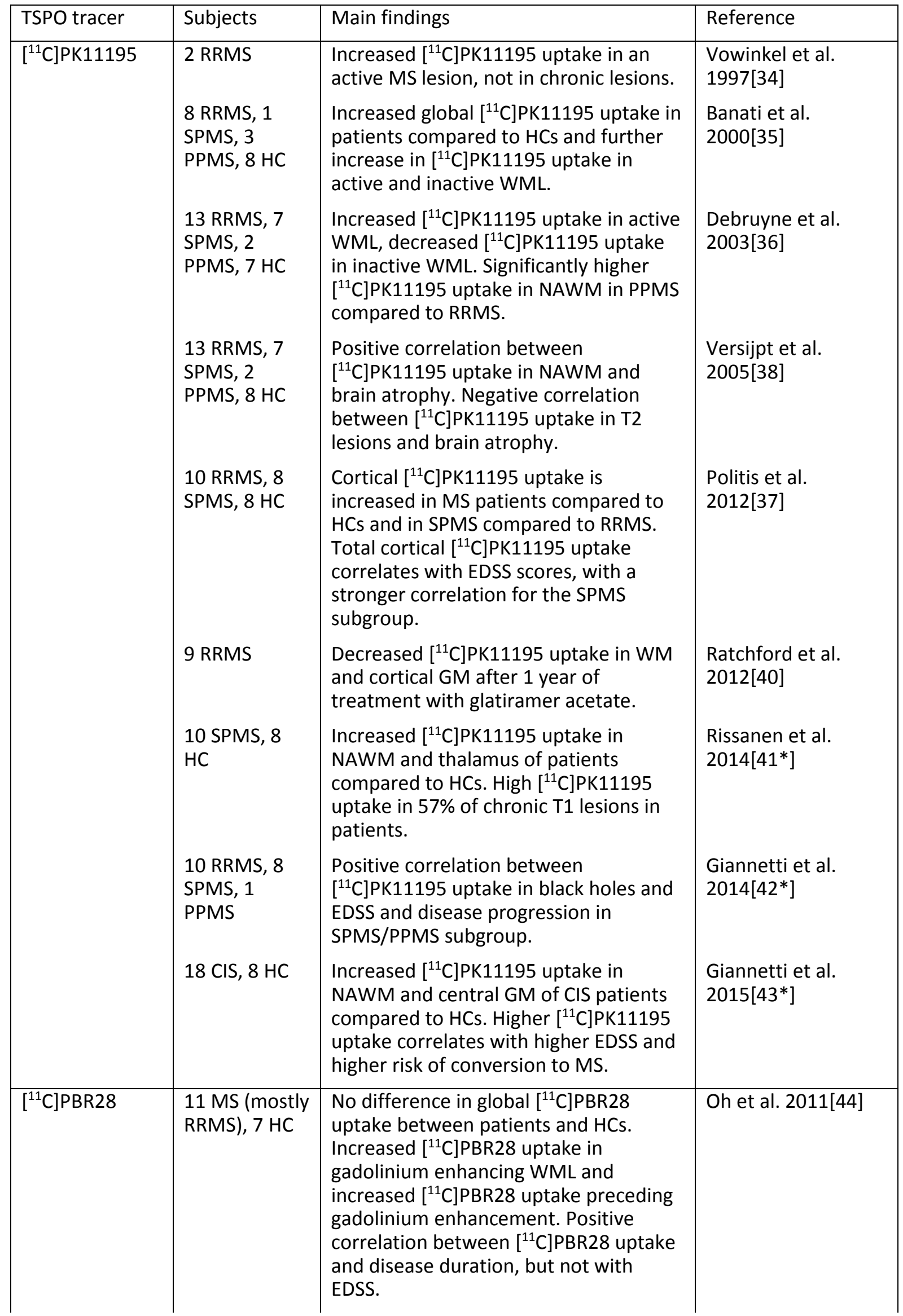




\begin{tabular}{|c|c|c|c|}
\hline & $\begin{array}{l}4 \text { stable } \\
\text { RRMS, } 4 \text { HCs }\end{array}$ & $\begin{array}{l}\text { No significant difference in }\left[{ }^{11} \mathrm{C}\right] \mathrm{PBR} 28 \\
\text { uptake between patients and HCs. Good } \\
\text { test-retest reproducibility. }\end{array}$ & $\begin{array}{l}\text { Park et al. } \\
2015\left[45^{*}\right]\end{array}$ \\
\hline$\left[{ }^{18} \mathrm{~F}\right]$ PBR111 & $\begin{array}{l}11 \text { RRMS, } 11 \\
\text { HC }\end{array}$ & $\begin{array}{l}\text { Increased }\left[{ }^{18} \mathrm{~F}\right] \mathrm{PBR} 111 \text { uptake in } \mathrm{WML} \\
\text { and perilesional WM compared to the } \\
\text { WM of HCs. Positive correlation } \\
\text { between }\left[{ }^{18} \mathrm{~F}\right] \mathrm{PBR} 111 \text { uptake and MS } \\
\text { severity scores in patients. }\end{array}$ & $\begin{array}{l}\text { Colasanti et al. } \\
2014\left[46^{*}\right]\end{array}$ \\
\hline$\left[{ }^{11} \mathrm{C}\right]$ vinpocetine & $\begin{array}{l}4 \text { MS (mostly } \\
\text { RRMS) }\end{array}$ & $\begin{array}{l}\text { Uptake of }\left[{ }^{11} \mathrm{C}\right] \text { vinpocetine is higher } \\
\text { than }\left[{ }^{11} \mathrm{C}\right] \mathrm{PK} 11195 \text { in regions of brain } \\
\text { damage, but with minimal overlap in } \\
\text { peak uptake regions. }\end{array}$ & Vas et al. 2008[47] \\
\hline$\left[{ }^{18}\right.$ F]FEDAA1106 & $9 \mathrm{RRMS}, 5 \mathrm{HC}$ & $\begin{array}{l}\text { No difference in overall }\left[{ }^{18} \mathrm{~F}\right] \mathrm{FEDAA} 1106 \\
\text { uptake between patients and controls. } \\
\text { Increase in }\left[{ }^{18} \mathrm{~F}\right] \mathrm{FEDAA} 1106 \text { uptake only } \\
\text { in one active MS lesions. (No } \\
\text { genotyping performed.) }\end{array}$ & $\begin{array}{l}\text { Takano et al. } \\
\text { 2013[48] }\end{array}$ \\
\hline$\left[{ }^{18} \mathrm{~F}\right] \mathrm{GE}-180-001$ & $9 \mathrm{RRMS}, 8 \mathrm{HC}$ & $\begin{array}{l}\text { Slighty increased }\left[{ }^{18} \mathrm{~F}\right] \mathrm{GE}-180-001 \\
\text { uptake in whole WM in patients } \\
\text { compared to HCs. Higher }\left[{ }^{18} \mathrm{~F}\right] \mathrm{GE}-180- \\
001 \text { uptake in inactive WML and a } \\
\text { further increase in gadolinium } \\
\text { enhancing lesions. (Preliminary data.) }\end{array}$ & Trigg et al. 2015[49] \\
\hline
\end{tabular}

